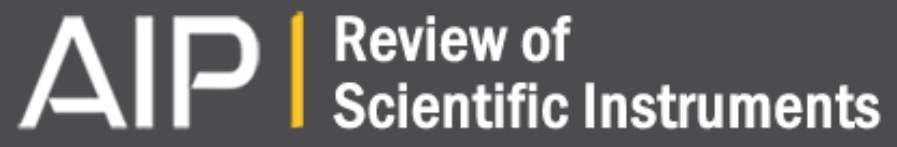

\section{Stokes-Doppler coherence imaging for ITER boundary tomography}

J. Howard, M. Kocan, S. Lisgo, and R. Reichle

Citation: Review of Scientific Instruments 87, 11E561 (2016); doi: 10.1063/1.4963712

View online: http://dx.doi.org/10.1063/1.4963712

View Table of Contents: http://scitation.aip.org/content/aip/journal/rsi/87/11?ver=pdfcov

Published by the AIP Publishing

\section{Articles you may be interested in}

Models, assumptions, and experimental tests of flows near boundaries in magnetized plasmas

Phys. Plasmas 23, 057101 (2016); 10.1063/1.4943523

Coherence imaging of scrape-off-layer and divertor impurity flows in the Mega Amp Spherical Tokamak (invited)a)

Rev. Sci. Instrum. 85, 11 D703 (2014); 10.1063/1.4891165

Comparison of edge turbulence imaging at two different poloidal locations in the scrape-off layer of Alcator CMod

Phys. Plasmas 20, 072503 (2013); 10.1063/1.4813758

Doppler coherence imaging and tomography of flows in tokamak plasmas (invited)a)

Rev. Sci. Instrum. 81, 10E528 (2010); 10.1063/1.3492422

The magnetic field structure of a snowflake divertor

Phys. Plasmas 15, 092501 (2008); 10.1063/1.2967900

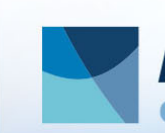




\title{
Stokes-Doppler coherence imaging for ITER boundary tomography
}

\author{
J. Howard, ${ }^{1, a)}$ M. Kocan, ${ }^{2}$ S. Lisgo, ${ }^{2}$ and R. Reichle ${ }^{2}$ \\ ${ }^{1}$ Plasma Research Laboratory, The Australian National University, Canberra, Australia \\ ${ }^{2}$ ITER Organization, Route de Vinon, F-13115 St. Paul lez, Durance, France
}

(Presented 8 June 2016; received 6 June 2016; accepted 14 September 2016; published online 24 October 2016)

An optical coherence imaging system is presently being designed for impurity transport studies and other applications on ITER. The wide variation in magnetic field strength and pitch angle (assumed known) across the field of view generates additional Zeeman-polarization-weighting information that can improve the reliability of tomographic reconstructions. Because background reflected light will be somewhat depolarized analysis of only the polarized fraction may be enough to provide a level of background suppression. We present the principles behind these ideas and some simulations that demonstrate how the approach might work on ITER. The views and opinions expressed herein do not necessarily reflect those of the ITER Organization. Published by AIP Publishing. [http://dx.doi.org/10.1063/1.4963712]

\section{INTRODUCTION}

Doppler coherence imaging of impurity ion flows and temperatures in the tokamak scrape-off-layer and divertor has been demonstrated successfully on the DIII-D and MAST tokamaks. ${ }^{1,2}$ Similar techniques have been applied to polarized multiplets to image the magnetic field pitch angle in tokamaks through the motional Stark effect. ${ }^{3-5}$ We combine these ideas to propose a Stokes-Doppler coherence-imaging camera to map the full Stokes vector of Zeeman-split impurity lines in the tokamak scrape-off-layer (SOL) and divertor.

Assuming the edge magnetic field is known, the variation of the splitting and polarization state of the Zeeman multiplet across the field of view can provide valuable additional Doppler tomographic information. Polarization filtering by a front-end polarimeter modifies the spectrum received by the interferometer. For appropriate choice of the interferometric delay (comparable to the optical coherence length of the multiplet) these changes are registered, along with the Doppler information, as modulations in the local interferometric fringe contrast and phase. ${ }^{6}$ On the other hand, because of its much shorter coherence length, the interferometer is insensitive to polarized broadband radiation falling within the prefilter passband. Because light that is diffusely scattered from rough surfaces such as divertor tiles becomes depolarized, Zeeman encoded coherence imaging offers the prospect of suppressing this contaminating component and providing additional information for the Doppler tomography of plasma edge and divertor flows.

In this paper, we describe the measurement principle and write down the projection formulas for the interferometrically measured Stokes parameters. Forward model projection images of the Stokes intensities for a simple emission phantom

Note: Contributed paper, published as part of the Proceedings of the 21st Topical Conference on High-Temperature Plasma Diagnostics, Madison, Wisconsin, USA, June 2016.

a)Electronic mail: john.howard@anu.edu.au and a standard ITER equilibrium are presented and future work is considered.

\section{DOPPLER IMAGING INTERFEROMETRY}

For Doppler broadened and shifted emission spectra, interferometric measurements of the optical coherence (fringe contrast and phase) deliver projections of the brightnessweighted ion temperature and flow speed. For a snapshot spatial-heterodyne imaging polarization interferometer, the brightness at position $(x, y)$ in the image plane is given by ${ }^{5,7}$

$$
\begin{aligned}
S & =\frac{I_{0}}{2}\left\{1 \pm \mathfrak{R}\left[\gamma\left(\phi_{0}\right) \exp \left(\mathrm{i} \phi_{0}\right)\right]\right\} \\
& =\frac{I_{0}}{2}\left[1+\zeta_{I} \zeta \cos \left(\phi_{I}+\phi_{0}+k_{0} x+\varphi\right)\right],
\end{aligned}
$$

where $I_{0}$ is the brightness and $\gamma\left(\phi_{0}\right) \exp \left(\mathrm{i} \phi_{0}\right)$ is the quasimonochromatic complex optical coherence. The phase delay offset $\phi_{0}=2 \pi L B\left(\lambda_{0}\right) / \lambda_{0}$ produced by a delay plate of thickness $L$ and birefringence $B$ is chosen to be comparable to the optical coherence length of the Doppler broadened line. For a spatially inhomogeneous plasma in local thermal equilibrium with temperature $T(\boldsymbol{r})$ and drift velocity $\boldsymbol{v}_{D}(\boldsymbol{r})$ (normalized to $c)$, the spectral line coherence is $\gamma\left(\phi_{0}\right)=g\left(\boldsymbol{r}, \hat{\phi}_{0}\right) \exp \left(\mathrm{i} \phi_{D}\right)$, where $g\left(\boldsymbol{r}, \hat{\phi}_{0}\right)=\exp \left[-T_{S}(\boldsymbol{r}) / T_{C}\right]$ is the Fourier transform of the Gaussian lineshape. The "characteristic temperature" is given by $k_{B} T_{C}=2 m_{S} c^{2} / \hat{\phi}_{0}^{2}$, where $m_{S}$ is the mass of the radiating species, and the group phase delay is $\hat{\phi}_{0}=\kappa \phi_{0}$ with constant $\kappa$ accounting for the chromatic dispersion of the birefringent crystal. ${ }^{6}$ Provided the Doppler phase shift $\phi_{D}=\hat{\phi}_{0} v_{D} . \hat{l}=\hat{\phi}_{0}\left(\Delta v_{D} / v_{0}\right)$, where $\Delta v_{D}$ is the Doppler optical frequency shift, is small, the interferometric quantities are ${ }^{6}$

$$
\begin{aligned}
& I_{0}=\int_{L} \varepsilon(\boldsymbol{r}) \mathrm{d} l, \\
& \zeta=\frac{1}{I_{0}} \int_{L} \varepsilon(\boldsymbol{r}) g(\boldsymbol{r}) \mathrm{d} l,
\end{aligned}
$$




$$
\varphi=\frac{\hat{\phi}_{0}}{I_{0} \zeta} \int_{L} \varepsilon(\boldsymbol{r}) g(\boldsymbol{r}) \boldsymbol{v}_{D} . \mathrm{d} \boldsymbol{l}
$$

The phase ramp $k_{0} x$ introduced by a shearing birefringent plate allows spatial heterodyne demodulation to recover the Doppler-produced perturbations $\zeta$ and $\varphi$ to the fringe contrast and phase. The instrumental fringe contrast and phase $\zeta_{\mathrm{I}}$ and $\phi_{\mathrm{I}}$ are determined by a calibration procedure (hereafter we take $\zeta_{I}=1$ and $\phi_{I}=0$ ).

\section{ZEEMAN SPECTRO-POLARIMETRY}

The Zeeman splitting and polarization of impurity ions have been used to measure the internal plasma magnetic field. ${ }^{8-10}$ Conversely, when the field is known, the polarization information can provide additional constraints for tomographic inversion. For simplicity, and because the fields in ITER are large (Paschen-Back effect), we assume a Zeeman triplet and choose a coordinate system where $\gamma$ is the angle between the magnetic field and the line-of-sight, and $\beta$ is the angle between the projection of $\boldsymbol{B}$ onto the $x-y$ imaging plane and the $x$ axis (Figure 1). In this case, the Stokes vectors for the $\pi$ and $\sigma$ Zeeman polarized components are given by ${ }^{6}$

$$
\begin{aligned}
\boldsymbol{s}_{\pi} & =\left(s_{\pi 0}, s_{1}, s_{2}, 0\right), \\
\boldsymbol{s}_{ \pm \sigma} & =\left(s_{ \pm \sigma 0},-s_{1},-s_{2}, \mp s_{3}\right) / 2, \\
\left(s_{1}, s_{2}, s_{3}\right) & \equiv \frac{1}{2}\left(\sin ^{2} \gamma \cos 2 \beta, \sin ^{2} \gamma \sin 2 \beta, 2 \cos \gamma\right) .
\end{aligned}
$$

The local Stokes components are fully polarized so that $s_{\pi 0}^{2}=s_{\pi 1}^{2}+s_{\pi 2}^{2}+s_{\pi 3}^{2}$ with similar conditions for the $\sigma$ terms. The zeroth components vary with view angle

$$
s_{\pi 0}(\gamma)=\frac{1}{2} \sin ^{2} \gamma, \quad s_{ \pm \sigma 0}(\gamma)=\frac{1}{2}\left(1+\cos ^{2} \gamma\right) .
$$

\section{A. Spherical quadrature polarimeter}

A spherical quadrature polarimeter based on the birefringent wave-plates of delay $\delta_{1}$ and $\delta_{2}$, and analyzer can be used to resolve the Stokes parameters. ${ }^{6}$ The response is

$$
\begin{aligned}
& P=\frac{I_{0}}{2} \boldsymbol{s} \cdot \boldsymbol{p}, \\
& \boldsymbol{p}=\left(1, \cos \delta_{2}, \sin \delta_{2} \sin \delta_{1}, \sin \delta_{2} \cos \delta_{1}\right),
\end{aligned}
$$

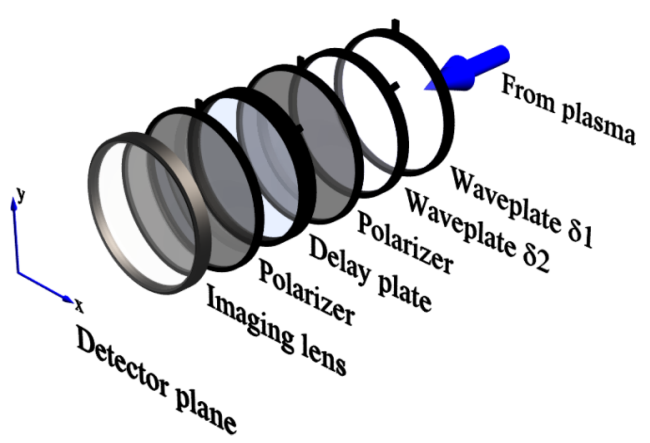

FIG. 1. Layout for the combined spherical quadrature polarimeter and interferometer. The polarimeter waveplates are mutually oriented at $45^{\circ}$ and introduce variable delays $\delta_{1}$ and $\delta_{2}$. The following interferometer with delay $\phi_{0}$ resolves the multiplet components. where $\boldsymbol{p}$ is analogous to the Stokes vector. Modulatable delays allow access to all of the Stokes parameters. For example, $\delta_{1}$ $=(0, \pi), \delta_{2}=0 \rightarrow \boldsymbol{p}=[1, \pm 1,0,0]$, and $\delta_{1}=0, \delta_{2}= \pm \pi / 2$, $\rightarrow \boldsymbol{p}=[1,0, \pm 1,0]$. By replacing the waveplates with Savart plates, it is possible to introduce angularly sheared optical phase delays which generate spatial heterodyne carrier fringe patterns in the focal plane of a final imaging lens. ${ }^{3}$ The polarimetric and interferometric functions can also be combined by introducing a bias plate in the polarimeter whose delay is comparable to the optical coherence length. For simplicity, we consider a modulatable polarimeter that allows each of the Stokes components to be isolated for subsequent interferometric analysis as shown in Fig. 1. It should be noted that the diagram does not show the necessary interference filter that spectrally isolates the Zeeman multiplet from other plasma visible emission.

\section{B. Stokes-Doppler tomography}

For an extended medium, the Stokes polarization components are integrated over the line of sight and summed over the multiplet components. Though the spectrally-integrated multiplet emission is nett unpolarized, the relative weights of the coherent parts of the $\pi$ and $\sigma$ interferograms depend on the interferometer phase delay. The total coherence of the $k$ th Stokes component is obtained by summing over $\pi$ and $\sigma$ lines

$$
\begin{aligned}
\gamma_{k}\left(\phi_{0}\right)= & \frac{1}{I_{0}} \int_{L} \mathrm{~d} l \varepsilon g\left\{\exp \left[\mathrm{i}\left(\phi_{D}-\phi_{B}\right)\right] s_{-\sigma k}\right. \\
& \left.+\exp \left(\mathrm{i} \phi_{D}\right) s_{\pi k}+\exp \left[\mathrm{i}\left(\phi_{D}+\phi_{B}\right)\right] s_{+\sigma k}\right\},
\end{aligned}
$$

where the phase shift $\phi_{B}=\hat{\phi}_{0} \Delta v_{B} / v_{0}$ is associated with the spectral shift $\Delta v_{B}=g_{L} e B / 4 \pi m_{\mathrm{e}}$ of the $\sigma$ manifold with Landé splitting factor $g_{L}$.

The contrast and phase for the $k$-th Stokes component interferogram $S_{k}$ [cf. Equation (2)] are then given by

$$
\begin{aligned}
\zeta_{k} & =\frac{1}{I_{0}} \int_{L} \varepsilon(\boldsymbol{r}) g(\boldsymbol{r}) w_{k}(\boldsymbol{r}) \mathrm{d} l \\
\varphi_{k} & =\frac{\hat{\phi}_{0}}{I_{0} \zeta_{k}} \int_{L} \varepsilon(\boldsymbol{r}) g(\boldsymbol{r}) w_{k}(\boldsymbol{r}) \boldsymbol{v}_{D} \cdot \mathrm{d} \boldsymbol{l},
\end{aligned}
$$

where we have taken $g_{\pi}=g_{ \pm \sigma}=g$ and the known spatially varying weight functions are given by

$$
\begin{aligned}
& w_{0}=\cos ^{2}\left(\phi_{B} / 2\right)-\cos ^{2} \gamma \sin ^{2}\left(\phi_{B} / 2\right), \\
& w_{1}=\cos 2 \beta \sin ^{2} \gamma \sin ^{2}\left(\phi_{B} / 2\right), \\
& w_{2}=\sin 2 \beta \sin ^{2} \gamma \sin ^{2}\left(\phi_{B} / 2\right), \\
& w_{3}=\sin \phi_{B} \cos \gamma .
\end{aligned}
$$

The zeroth Stokes component interferogram $S_{0}$ has intensity $I_{0}$. Because the Zeeman multiplet is nett unpolarized, the summed "intensities" for the polarized components are zero $\left(I_{k}=0, k=1,2,3\right)$. The weight function $w_{0}$ represents the contrast change associated with the orientationdependent relative intensity of the shifted $\sigma$ and unshifted $\pi$ components. 


\section{Divertor and SOL brightness model}

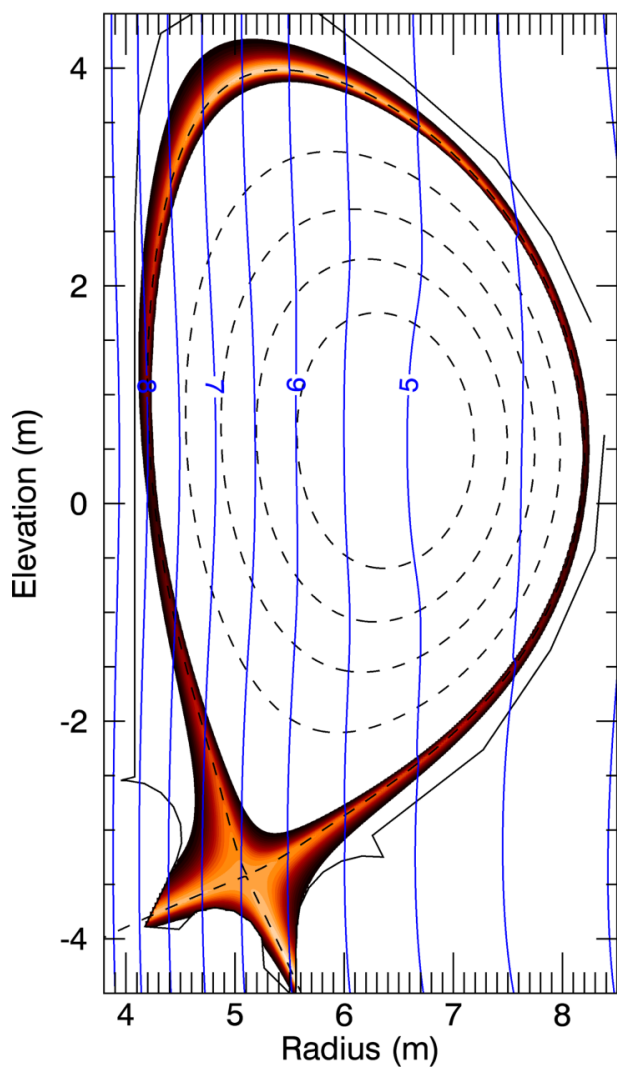

FIG. 2. Simple brightness model used for projection simulations. Flux surface and field strength contours are superimposed.

\section{ITER FORWARD MODEL}

A model of the divertor view for equatorial port \#8 has been developed to assess the potential of Stokes-Doppler imaging for improving the tomography and/or helping to discriminate against background reflections. We use a standard
ITER equilibrium with the plasma radiating in a narrow region in the SOL and divertor as shown in Fig. 2.

The small Doppler phase condition $\phi_{D}<1$ constrains the utility of the Zeeman weighting through the ratio $\rho$ $=\phi_{B} / \phi_{D}=\Delta v_{B} / \Delta v_{D}$ which scales as $\rho \sim B \lambda_{0} / v_{D}$. To illustrate, we take singly ionized helium at $\sim 50 \mathrm{eV}$ and assume $v_{D} \sim v_{\text {thi }}$. For SOL emission at $468 \mathrm{~nm}$, we find $0.5<\rho<1$ for field strength from $4 \mathrm{~T}$ at the outside edge to $8 \mathrm{~T}$ on the inside, so the weights $w_{k}$ vary substantially across the view.

For the simulations we calculate the contrast images $\zeta_{k}$ with delay $\hat{\phi}_{0}$ chosen such that $0.5<\phi_{B}<1$ across the plasma, and ignore the ion temperature $(g=1)$ in order to visualize the effects of the Zeeman weighting and assess the degree of polarization given by $\left(S_{1}^{2}+S_{2}^{2}+S_{3}^{2}\right)^{1 / 2} / S_{0}$ (Fig. 3). The angular width of the field of view is $65^{\circ}$, and for reference, the computed images are superimposed on a semi-transparent simplified model of the ITER vacuum vessel and divertor structure. Images of the interferometric phase depend on the details of the chosen flow model and will be considered in a later paper.

It is seen that the degree of polarization is greatest at the inside SOL where the Zeeman splitting is largest. On the other hand, the contrast $\zeta_{0}$ is least in this region because of the beating of the $\sigma$ component interferograms. The remaining Stokes images are coded by structural information about the magnetic field in the region illuminated by the emission model. It is clear that all components carry significant weight, though the tangential nature of the view and the $\sin ^{2}\left(\phi_{B} / 2\right)$ weighting reduces $S_{1}$ and $S_{2}$ compared with the $S_{3}$ component. In the high magnetic fields of ITER, it is important to consider the full polarized multiplet when interpreting images of the optical coherence. When the field is known, this information could improve tomographic reconstructions and help discriminate against partially depolarized wall reflections. At the very least, tomography of the polarized fraction only, will suppress the contributions from both polarized and unpolarized broadband emission that enters the filter passband.
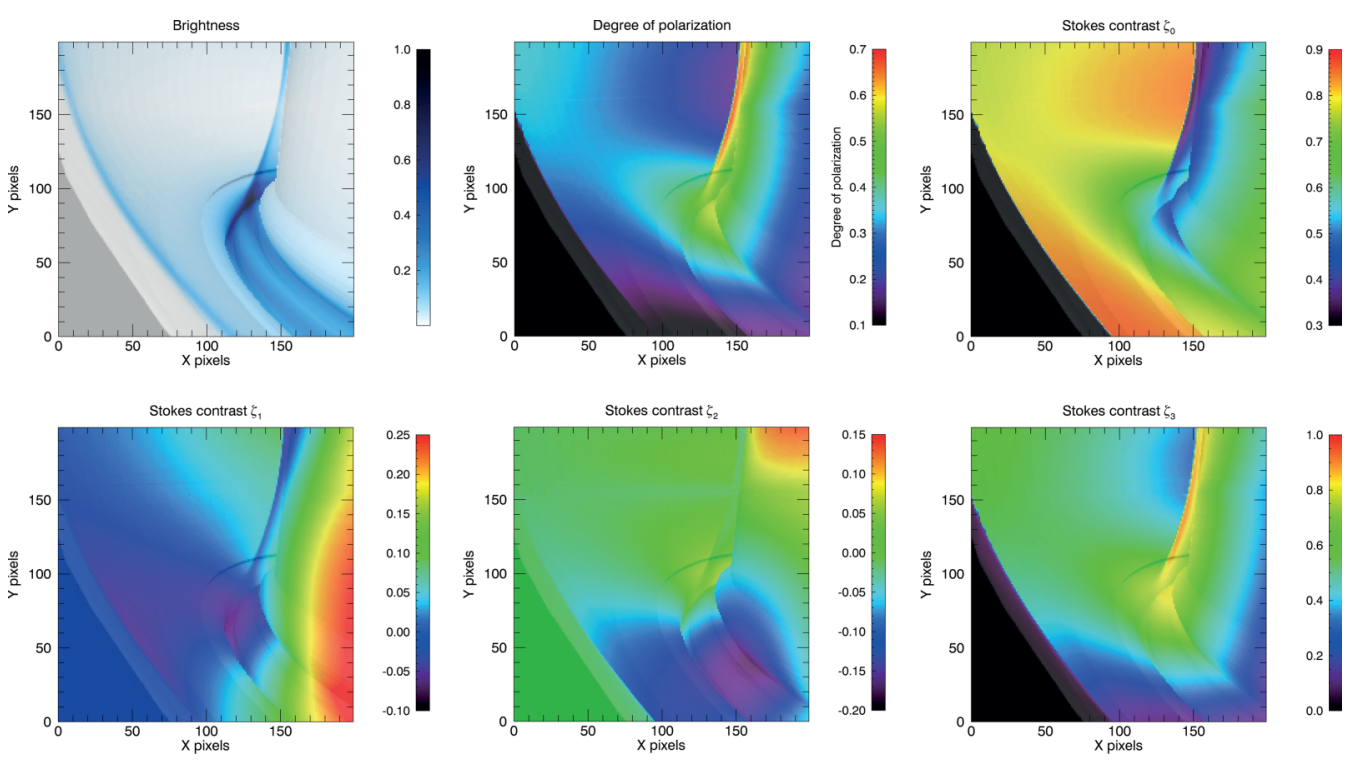

FIG. 3. Left to right and top to bottom: Projected brightness, the degree of polarization and images of the Stokes component contrasts. See text for discussion. 
${ }^{1}$ J. Howard, A. Diallo, M. Creese, B. Blackwell, S. L. Allen, R. M. Ellis, G. D. Porter, W. Meyer, M. E. Fenstermacher, N. H. Brooks, M. Van Zeeland, and R. L. Boivin, Rev. Sci. Instrum. 81, 10E528 (2010).

${ }^{2}$ S. A. Silburn, J. R. Harrison, J. Howard, K. J. Gibson, H. Meyer, C. A. Michael, and R. M. Sharples, Rev. Sci. Instrum. 85, 11D703 (2014).

${ }^{3}$ J. Howard, Plasma Phys. Controlled Fusion 50, 125003 (2008).

${ }^{4}$ O. Ford, J. Howard, M. Reich, J. Hobirk, J. Svensson, and R. Wolf, in Proceedings 40th European Physical Society Conference on Plasma Physics, O2 (European Physical Society, 2013).

5 J. Howard, C. Michael, H. Chen, R. Lester, A. Thorman, and J. Chung, J. Instrum. 10, P09023 (2015).
${ }^{6}$ J. Howard, C. Michael, F. Glass, and A. Danielsson, Plasma Phys. Controlled Fusion 45, 1143 (2003).

7J. Howard, J. Phys. B: At., Mol. Opt. Phys. 43, 144010 (2010).

${ }^{8}$ U. Feldman, J. F. Seeley, N. R. J. Sheeley, S. Suskewer, and A. M. Title, J. Appl. Phys. 56, 2512 (1984).

${ }^{9}$ H. Kuramoto, N. Hiraki, S. Moriyama, K. Toi, K. Sata, and K. Narihara, Fusion Eng. Des. 34-35, 285 (1997).

${ }^{10}$ D. Thomas, A. Bozek, J. Robinson, K. Burrell, T. Carlstrom, T. Osborne, R. Snider, D. Finkenthal, R. Jayakumar, M. Makowski, D. Nilson, and B. Rice, Rev. Sci. Instrum. 72, 1023 (2001). 